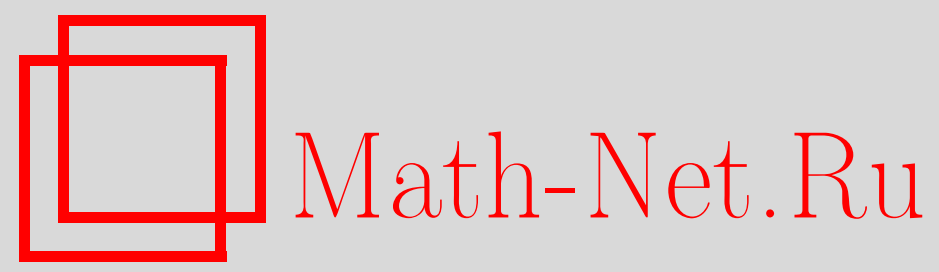

B.

Roos,

Multinomial and Krawtchouk Approximations to the Generalized Multinomial Distribution, Теория вероятн. и ее примен., 2001, том 46, выпуск 1, 117-133

DOI: https://doi.org/10.4213/tvp3954

Использование Общероссийского математического портала MathNet.Ru подразумевает, что вы прочитали и согласны с пользовательским соглашением

http://www. mathnet.ru/rus/agreement

Параметры загрузки:

IP : 54.237 .59 .107

26 апреля 2023 г., 13:05:36

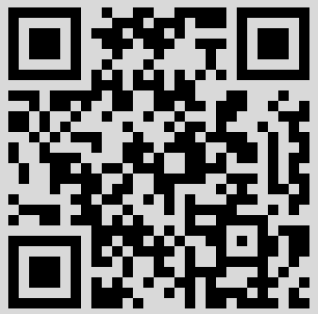




\section{MULTINOMIAL AND KRAWTCHOUK APPROXIMATIONS TO THE GENERALIZED MULTINOMIAL DISTRIBUTION}

Изучается аппроксимация обобщенного полиномиального распределения полиномиальными распределениями и конечными мерами со знаком, определяемыми по соответствующему многомерному разложению Кравчука. Даны границы для нормы полной вариации и $\ell_{\infty}$-нормы. Используемый метод есть многомерное обобщение метода [6].

Ключевые слова и фразы: приближение полиномами, обобщенное полиномиальное распределение, многомерное разложение Кравчука, меры со знаком, норма полной вариации, $\ell_{\infty}$-норма.

\section{Introduction}

1.1. The problem. Let $S_{n}$ be the sum of independent Bernoulli random vectors $X_{1}, \ldots, X_{n}$ in $\mathbf{R}^{k}$ with probabilities

$$
\mathbf{P}\left\{X_{j}=e_{r}\right\}=p_{j, r}, \quad \mathbf{P}\left\{X_{j}=0\right\}=1-\sum_{r=1}^{k} p_{j, r}=p_{j, 0}
$$

for $j \in\{1, \ldots, n\}$ and $r \in\{1, \ldots, k\}$, where $e_{r} \in \mathbf{R}^{k}$ is the vector with entry 1 at position $r$ and 0 otherwise.

In this paper, we consider the approximation of the generalized multinomial distribution $P^{S_{n}}$ by multinomial distributions and also by finite signed measures resulting from the corresponding multivariate Krawtchouk expansion. Such approximations of $P^{S_{n}}$ are useful, for example, in case of $X_{1}, \ldots, X_{n}$ being nearly identically distributed. The main task is to give some bounds for the approximation errors with respect to the total variation norm and the $\ell_{\infty}$-norm.

Using Stein's method, Loh [5, Theorem 5] has recently provided a somewhat complicated upper bound for the total variation distance between $P^{S_{n}}$ and a multinomial distribution. In the univariate case $k=1$, the problem reduces to the binomial approximation of the Poisson binomial distribution.

*Institut für Mathematische Stochastik, Universität Hamburg, Bundesstraße 55, D-20146 Hamburg, Germany; e-mail: roos@math.uni-hamburg.de 
It follows from Ehm's [3] results (see (1) below) that, in this case, Loh's [5, Corollary 3] bound has not the correct order.

The method of the present paper is a multivariate extension of that in Roos [6]. Further, some ideas of Shorgin [9] and Roos [7] are used. We have to deal with the multivariate generalizations of the Krawtchouk polynomials. A complication is that these are, contrary to the univariate case $k=1$, no longer orthogonal with respect to the multinomial distribution (see (4), (5), and the remarks thereafter). For the binomial approximation in the univariate case, our upper bound with respect to the total variation norm has the correct order (see Corollary 1 and formula (1)).

For Poisson approximations of $P^{S_{n}}$ see, for example, Barbour [1], Deheuvels and Pfeifer [2], Roos [7], [8], and the references therein.

1.2. Further notation. We always assume that $m \in \mathbf{Z}_{+}, u, \tilde{u}, v, \tilde{v}$, $w \in \mathbf{Z}_{+}^{k}$, and $y, z \in \mathbf{C}^{k}$. We use the standard multi-index notation: For $z=\left(z_{1}, \ldots, z_{k}\right)$ and $u=\left(u_{1}, \ldots, u_{k}\right)$, we set $z^{u}=z_{1}^{u_{1}} \cdots z_{k}^{u_{k}},|u|=\sum_{r=1}^{k} u_{r}$, and $u !=u_{1} ! \cdots u_{k}$ !. We always let $0^{0}=1$. We write $u \leqslant v$ in the case that $u_{r} \leqslant v_{r}$ for all $r$; if additionally at least one of these inequalities is strict, we write $u<v$. Similarly, $u \wedge v \in \mathbf{Z}_{+}^{k}$ is the vector with $r$ th component $\min \left\{u_{r}, v_{r}\right\}$. All of our series and sums are carried out over subsets of $\mathbf{Z}_{+}^{k}$, unless otherwise specified. For example, the sign $\sum_{|u| \leqslant m}$ means the sum over all $u \in \mathbf{Z}_{+}^{k}$ with $|u| \leqslant m$.

Let $q=\left(q_{1}, \ldots, q_{k}\right) \in(0,1)^{k}$ with $q_{0}=1-\sum_{r=1}^{k} q_{r} \in(0,1)$, and, for $r \in\{0, \ldots, k\}$,

$\bar{p}_{r}=\frac{1}{n} \sum_{j=1}^{n} p_{j, r} \in(0,1), \quad \bar{p}=\left(\bar{p}_{1}, \ldots, \bar{p}_{k}\right), \quad \gamma_{m}(r, q)=\sum_{j=1}^{n}\left(q_{r}-p_{j, r}\right)^{m}$

Note that $\bar{p}_{0}=1-\sum_{r=1}^{k} \bar{p}_{r}$ and that $\gamma_{1}(r, \bar{p})=0$ for all $r \in\{0, \ldots, k\}$. The counting density $M(\cdot, m, q)$ of the multinomial distribution $\mathscr{M}(m, q)$ is given by

$$
M(w, m, q)=\mathscr{M}(m, q)(\{w\})= \begin{cases}\frac{m !}{w !(m-|w|) !} q^{w} q_{0}^{m-|w|} & \text { if }|w| \leqslant m \\ 0 & \text { otherwise }\end{cases}
$$

In the case $k=1, \mathscr{M}(m, q)$ is a binomial distribution, which is also denoted by $\mathscr{B}(m, q)$. For $f: \mathbf{Z}_{+}^{k} \rightarrow \mathbf{R}$ and $r \in\{1, \ldots, k\}$, let $\Delta_{r} f: \mathbf{Z}_{+}^{k} \rightarrow \mathbf{R}$ with

$$
\left(\Delta_{r} f\right)(w)= \begin{cases}f\left(w-e_{r}\right)-f(w) & \text { if } w_{r} \geqslant 1 \\ -f(w) & \text { if } w_{r}=0\end{cases}
$$

Further, let $\Delta_{r}^{0} f=f$ and $\Delta_{r}^{m} f=\Delta_{r}^{m-1}\left(\Delta_{r} f\right)$ for $m \in \mathbf{N}$. Clearly, $\Delta_{r} \Delta_{s} f=\Delta_{s} \Delta_{r} f$ for $r, s \in\{1, \ldots, k\}$. Let $\Delta^{u} f=\Delta_{1}^{u_{1}} \cdots \Delta_{k}^{u_{k}} f$. We 
set $\Delta^{u} M(w, m, q)=\left(\Delta^{u} M(\cdot, m, q)\right)(w)$. Let $\|f\|_{1}=\sum_{w \in \mathbf{Z}_{+}^{k}}|f(w)|$ and $\|f\|_{\infty}=\sup _{w \in \mathbf{Z}_{+}^{k}}|f(w)|$. For a finite signed measure $Q$ concentrated on $\mathbf{Z}_{+}^{k}$ with counting density $f_{Q}$, let $\|Q\|_{1}=\left\|f_{Q}\right\|_{1}$ and $\|Q\|_{\infty}=\left\|f_{Q}\right\|_{\infty}$. For $x \in \mathbf{C}$, let $\left(\begin{array}{c}x \\ m\end{array}\right)=\prod_{j=1}^{m}(x-j+1) / j$.

1.3. Some known facts in the univariate case. In this subsection, let $k=1$. Using the Stein-Chen method, Ehm [3, Theorem 1, Lemma 2] proved the following estimates:

$$
\begin{aligned}
\frac{\gamma_{2}(1, \bar{p})}{62} \min \left\{\frac{1}{n \bar{p}_{1} \bar{p}_{0}}, 1\right\} & \leqslant\left\|P^{S_{n}}-\mathscr{B}\left(n, \bar{p}_{1}\right)\right\|_{1} \\
& \leqslant 2 \gamma_{2}(1, \bar{p}) \min \left\{\frac{1}{n \bar{p}_{1} \bar{p}_{0}}, 1\right\} .
\end{aligned}
$$

Hence the term on the right-hand side of (1) and $\left\|P^{S_{n}}-\mathscr{B}\left(n, \bar{p}_{1}\right)\right\|_{1}$ have the same order; further, this distance is small if and only if $\gamma_{2}(1, \bar{p})\left(n \bar{p}_{1} \bar{p}_{0}\right)^{-1}$ is small (see Ehm [3, Corollary 2]). A generalization of this fact for $k \in \mathbf{N}$ being fixed or bounded is shown in Corollary 2 of the present paper.

For further results on the univariate case, see Roos [6] and the references therein.

\section{Main results}

2.1. The Krawtchouk expansion of $P^{S_{n}}$.

Theorem 1. For arbitrary $q$,

$$
\mathbf{P}\left\{S_{n}=w\right\}=\sum_{|u| \leqslant n} a_{u}(q) \Delta^{u} M(w, n-|u|, q),
$$

where the coefficients $a_{u}(q)$ are defined by the relation

$$
\sum_{|u| \leqslant n} a_{u}(q) z^{u}=\prod_{j=1}^{n}\left(1+\sum_{r=1}^{k}\left(p_{j, r}-q_{r}\right) z_{r}\right) .
$$

In this paper, we call the right-hand side of (2) the Krawtchouk expansion of $P^{S_{n}}$ with parameter $q$; further, we call the coefficients $a_{u}(q)$ the Krawtchouk coefficients of $P^{S_{n}}$ with parameter $q$. For the approximation of $P^{S_{n}}$, we use the finite signed measures $\mathscr{M}_{t}(n, q)(t \in\{0, \ldots, n\})$ concentrated on $\mathbf{Z}_{+}^{k}$ with counting density

$$
\mathscr{M}_{t}(n, q)(\{w\})=\sum_{|u| \leqslant t} a_{u}(q) \Delta^{u} M(w, n-|u|, q) .
$$

R e m a r k s. 1 . For $t \geqslant 1, \mathscr{M}_{t}(n, q)$ depends not only on $n$ and $q$ but also on the $p_{j, r}$. 
2. For all $t$, we have $\mathscr{M}_{t}(n, q)\left(\mathbf{Z}_{+}^{k}\right)=1$ and $\mathscr{M}_{t}(n, q)(\{w\})=0$ in the case $|w|>n$.

The Krawtchouk expansion of $P^{S_{n}}$ and its coefficients are associated with two sets $\mathscr{K}_{m, q}=\left\{K_{u}(z, m, q)|| u \mid \leqslant m\right\}$ and $\widetilde{\mathscr{K}}_{m, q}=\left\{\widetilde{K}_{u}(z, m, q)|| u \mid \leqslant\right.$ $m\}$ of multivariate Krawtchouk polynomials with variable $z$, which are, for $|u| \leqslant m$, defined by

$$
\begin{aligned}
& K_{u}(z, m, q)=\sum_{v \leqslant u}\left(\begin{array}{c}
m-\sum_{r=1}^{k} z_{r} \\
|u-v|
\end{array}\right) \frac{|u-v| !(-q)^{u-v} q_{0}^{|v|}}{(u-v) !} \prod_{r=1}^{k}\left(\begin{array}{c}
z_{r} \\
v_{r}
\end{array}\right), \\
& \widetilde{K}_{u}(z, m, q)=\sum_{v \leqslant u} \frac{(m-|v|) !(-q)^{u-v}}{(u-v) !(m-|u|) !} \prod_{r=1}^{k}\left(\begin{array}{c}
z_{r} \\
v_{r}
\end{array}\right) .
\end{aligned}
$$

R e m a r k s. 1. Apparently Griffiths [4] is the first author who treated a multivariate generalization of the Krawtchouk polynomials, though he did not give an explicit formula comparable with (4) or (5). For $k=2$, another version of the Krawtchouk expansion and the two kinds of Krawtchouk polynomials above can also be found in Takeuchi and Takemura [11, Section 3], who considered various approximations of the counting density and the distribution function of the sum of not necessarily independent Bernoulli random vectors. The multivariate Krawtchouk polynomials for arbitrary $k$ were given by Tratnik [12, formulas (3.3) and (3.4)] by means of the generalized Kampé de Fériet hypergeometric series. However, we use our notation, since they seem to be more convenient for our purposes. Note that in formula (3.4) of Tratnik [12] the « $x_{1} »$ must be replaced with «- $x_{1} 》$.

2. In the case that $k=1$ and $u \leqslant m, K_{u}(z, m, q)$ coincides with $\widetilde{K}_{u}(z, m, q)$ and also with the classical univariate Krawtchouk polynomial of $u$ th degree and parameters $m$ and $q$ (see (22), (23), and, for example, Szegö $[10$, formula $(2.82 .2)])$.

3. Suppose $m \geqslant 1$.

(a) The elements of $\mathscr{K}_{m, q}$ constitute a system of orthogonal polynomials with respect to the multinomial distribution $\mathscr{M}(m, q)$ if and only if $k=1$ (see (26)).

(b) The elements of $\mathscr{K}_{m, q}$ and $\widetilde{\mathscr{K}}_{m, q}$ constitute a biorthogonal system with respect to the multinomial distribution $\mathscr{M}(m, q)$ (see Tratnik [12, formula (3.9)] or (27)).

4. There is a formula for $\Delta^{u} M(w, n-|u|, q)$ (respectively for $\left.a_{u}(q)\right)$, containing the term $K_{u}(w, n, q)$ (respectively the terms $\widetilde{K}_{u}(w, n, q)$ for $|w| \leqslant n$ ) (see (21) and (7)).

In the following proposition, we provide some alternative formulae for the Krawtchouk coefficients of $P^{S_{n}}$ with parameter $q$. Let $\mu_{(u)}$ be the $u$ th 
factorial moment of $S_{n}$, defined by

$$
\mu_{(u)}=\sum_{w \in \mathbf{Z}_{+}^{k}} \mathbf{P}\left\{S_{n}=w\right\} \prod_{r=1}^{k}\left[\left(\begin{array}{c}
w_{r} \\
u_{r}
\end{array}\right) u_{r} !\right]
$$

Proposition 1. If $1 \leqslant|u| \leqslant n$ and $R \in(0, \infty)^{k}$, then

$$
\begin{aligned}
a_{u}(q) & =\sum_{|w| \leqslant n} \mathbf{P}\left\{S_{n}=w\right\} \widetilde{K}_{u}(w, n, q) \\
& =-\frac{1}{|u|} \sum_{v<u} \frac{|u-v| !}{(u-v) !}\left(\sum_{j=1}^{n} \prod_{r=1}^{k}\left(q_{r}-p_{j, r}\right)^{u_{r}-v_{r}}\right) a_{v}(q) \\
& =\sum_{v \leqslant u} \frac{(n-|v|) !(-q)^{u-v} \mu_{(v)}}{(u-v) !(n-|u|) ! v !} \\
& =\frac{1}{(2 \pi)^{k} R^{u}} \int_{0}^{2 \pi} \cdots \int_{0}^{2 \pi} \exp \left(-i \sum_{r=1}^{k} u_{r} x_{r}\right) \\
& \times \prod_{j=1}^{n}\left(1+\sum_{r=1}^{k}\left(p_{j, r}-q_{r}\right) R_{r} e^{i x_{r}}\right) d x_{1} \cdots d x_{k} .
\end{aligned}
$$

$\mathrm{R}$ e $\mathrm{m}$ a $\mathrm{r} \mathrm{k}$. We have $a_{0}(q)=1$ and, for $r, s \in\{1, \ldots, k\}$ with $r \neq s$,

$$
\begin{aligned}
a_{e_{r}}(q) & =-\gamma_{1}(r, q) \\
a_{e_{r}+e_{s}}(q) & =\gamma_{1}(r, q) \gamma_{1}(s, q)-\sum_{j=1}^{n}\left(q_{r}-p_{j, r}\right)\left(q_{s}-p_{j, s}\right), \\
a_{2 e_{r}}(q) & =\frac{1}{2}\left(\gamma_{1}^{2}(r, q)-\gamma_{2}(r, q)\right) .
\end{aligned}
$$

Hence $\mathscr{M}_{0}(n, q)=\mathscr{M}(n, q)$ and

$$
\mathscr{M}_{1}(n, q)(\{w\})=M(w, n, q)\left[1-\sum_{r=1}^{k} \frac{\gamma_{1}(r, q)}{n q_{r} q_{0}}\left(q_{r}|w|+q_{0} w_{r}-n q_{r}\right)\right] .
$$

Observe that $\mathscr{M}_{1}(n, \bar{p})=\mathscr{M}(n, \bar{p})$. For $t \geqslant 2, \mathscr{M}_{t}(n, q)(\{w\})$ can be evaluated by using (4), (8), and (21).

2.2. The bounds for the distances. We use the following notation. For $r \in\{1, \ldots, k\}$, let

$$
\eta(r, q)=2 \gamma_{2}(r, q)+\gamma_{1}^{2}(r, q),
$$

$\delta(r, q)=\eta(r, q) \min \left\{\frac{1}{2 n q_{r} q_{0}}, \frac{2}{e}\right\}, \quad \zeta(r, q)=\eta(r, q) \min \left\{\frac{1}{2 n q_{r} q_{0}}, \frac{2 e}{3}\right\}$. 
Theorem 2. Let $t \in\{0, \ldots, n-1\}$. If $\sum_{r=1}^{k} \sqrt{\delta(r, q)}<e^{-1 / 2}$, then

$$
\left\|P^{S_{n}}-\mathscr{M}_{t}(n, q)\right\|_{1} \leqslant \frac{e^{(t+1) / 2}\left(\sum_{r=1}^{k} \sqrt{\delta(r, q)}\right)^{t+1}}{1-\sum_{r=1}^{k} \sqrt{e \delta(r, q)}} .
$$

$\mathrm{R}$ e $\mathrm{m}$ a $\mathrm{rk}$. The proof of Theorem 2 will also show that

$$
\left\|\mathscr{M}_{t}(n, q)\right\|_{1} \leqslant \sum_{j=0}^{t}\left(\sum_{r=1}^{k} \sqrt{e \delta(r, q)}\right)^{j} .
$$

Further, inequality (15) remains valid, if $t \geqslant 1, q=\bar{p}$, and the summand for $j=1$ in the first sum on the right-hand side is omitted.

The following corollary shows that it is possible to remove the singularity on the right-hand side of (14).

Corollary 1. Let $t \in\{0, \ldots, n-1\}$. Then

$$
\left\|P^{S_{n}}-\mathscr{M}_{t}(n, q)\right\|_{1} \leqslant c_{1}(t) e^{(t+1) / 2}\left(\sum_{r=1}^{k} \sqrt{\delta(r, q)}\right)^{t+1},
$$

where $c_{1}(t)=\left(1-x_{t}\right)^{-1} \leqslant 2 t+3$ and $x_{t}$ is the unique positive solution of the equation $x^{t+1}+x / 2=1$. Relation (16) remains valid, if $t \geqslant 1, q=\bar{p}$, and $c_{1}(t)$ is replaced by

$$
\tilde{c}_{1}(t)=\frac{1}{1-\tilde{x}_{t}} \leqslant \frac{2 t-1}{t+1-\sqrt{t^{2}+2}},
$$

where $\widetilde{x}_{t}$ is the unique positive solution of the equation $\widetilde{x}^{t+1}-\widetilde{x}^{2} / 2+\widetilde{x}=1$.

$\mathrm{R}$ e m a r k s. 1 . We have $\tilde{c}_{1}(t)<c_{1}(t)$ for $t \geqslant 1$. The inequality given for $c_{1}(t)$ (respectively for $\widetilde{c}_{1}(t)$ ) is an equality, if $t=0$ (respectively if $t=1$ ).

2. In view of $(1)$, we see that

$$
\left\|P^{S_{n}}-\mathscr{M}(n, \bar{p})\right\|_{1} \geqslant \frac{1}{62} \max _{0 \leqslant r \leqslant k}\left(\gamma_{2}(r, \bar{p}) \min \left\{\frac{1}{n \bar{p}_{r}\left(1-\bar{p}_{r}\right)}, 1\right\}\right) .
$$

Further, in the case $k=1, t=1$, and $q=\bar{p}$, the bound in (16) has the correct order; under these assumptions, the next corollary coincides with Corollary 2 of Ehm [3] (see Subsection 1.3 of the present paper).

In what follows, we write $S_{n}=\left(S_{n}(1), \ldots, S_{n}(k)\right)$ and set $S_{n}(0)=$ $n-\sum_{r=1}^{k} S_{n}(r)$.

Corollary 2. In the case of fixed or bounded dimension $k$, the distance $\left\|P^{S_{n}}-\mathscr{M}(n, \bar{p})\right\|_{1}$ is small if and only if

$$
\sum_{r=0}^{k} \frac{\gamma_{2}(r, \bar{p})}{n \bar{p}_{r}\left(1-\bar{p}_{r}\right)}=\sum_{r=0}^{k}\left[1-\frac{\mathbf{D} S_{n}(r)}{n \bar{p}_{r}\left(1-\bar{p}_{r}\right)}\right]
$$

is small. 
The following proposition shows that sometimes $\left\|P^{S_{n}}-\mathscr{M}(n, \bar{p})\right\|_{1}$ can be estimated by using results for the univariate case.

Proposition 2. If $p_{j, r}=b_{j} b_{r}^{\prime}$ with $b_{j}, b_{r}^{\prime} \in[0, \infty)$, for $j \in\{1, \ldots, n\}$, $r \in\{1, \ldots, k\}$, and if $T_{n}=\sum_{r=1}^{k} S_{n}(r)$, then

$$
\left\|P^{S_{n}}-\mathscr{M}(n, \bar{p})\right\|_{1}=\left\|P^{T_{n}}-\mathscr{B}\left(n, \sum_{r=1}^{k} \bar{p}_{r}\right)\right\|_{1} .
$$

R e m a r k s. 1. Under the assumptions of Proposition 2, Ehm's [3] results (see (1)) lead to the inequalities.

$$
\frac{1}{62} \xi \leqslant\left\|P^{S_{n}}-\mathscr{M}(n, \bar{p})\right\|_{1} \leqslant 2 \xi
$$

where

$$
\xi=\left[\sum_{j=1}^{n}\left(\sum_{r=1}^{k} \bar{p}_{r}-\sum_{r=1}^{k} p_{j, r}\right)^{2}\right] \min \left\{\frac{1}{n\left(\sum_{r=1}^{k} \bar{p}_{r}\right) \bar{p}_{0}}, 1\right\}
$$

2. From Corollary 1 it follows that

$$
\left\|P^{S_{n}}-\mathscr{M}(n, \bar{p})\right\|_{1} \leqslant c k^{\alpha} \sum_{r=1}^{k} \gamma_{2}(r, \bar{p}) \min \left\{\frac{1}{n \bar{p}_{r} \bar{p}_{0}}, 1\right\}
$$

with $c=4 /(2-\sqrt{3})$ and $\alpha=1$. But generally, (18) cannot hold with absolute constants $c \in(0, \infty)$ and $\alpha \in[0,1)$. To verify this assertion, we assume that the conditions in Proposition 2 hold and let $n \in\{2,3, \ldots\}$, $k=n, b_{j}=\left(j n \sum_{m=1}^{n} 1 / m\right)^{-1}$ for $j \in\{1, \ldots, n\}$, and $b_{1}^{\prime}=\cdots=b_{k}^{\prime}=1$. Using the notation of Remark 1 , we then have

$$
\xi=\frac{\sum_{j=1}^{n} 1 / j^{2}}{\left(\sum_{j=1}^{n} 1 / j\right)^{2}}-\frac{1}{n}
$$

whereas $c \xi / n^{1-\alpha}$ is the value of the right-hand side of (18).

Theorem 3. Let $t \in\{0, \ldots, n-1\}, c_{2}=(2 \sqrt{3})^{-1}(1+\sqrt{\pi / 2})$. If $\sum_{r=1}^{k} \sqrt{\zeta(r, q)}<3^{-1 / 2}$, then

$$
\begin{aligned}
\left\|P^{S_{n}}-\mathscr{M}_{t}(n, q)\right\|_{\infty} \leqslant & 3^{(t+1) / 2} c_{2}^{k}\left(\prod_{r=1}^{k} \min \left\{\frac{1}{n q_{r} q_{0}}, \frac{4 e}{3}\right\}\right)^{1 / 2} \\
& \times \frac{\left(\sum_{r=1}^{k} \sqrt{\zeta(r, q))^{t+1}}\right.}{1-\sum_{r=1}^{k} \sqrt{3 \zeta(r, q)}}
\end{aligned}
$$




\section{Proofs}

$\mathrm{Pr}$ o of of $\mathrm{Th}$ e or e m 1. Inductively it is easy to show that

$$
\sum_{w \in \mathbf{Z}_{+}^{k}} \Delta^{u} M(w, m, q) z^{w}=\left(1+\sum_{r=1}^{k} q_{r}\left(z_{r}-1\right)\right)^{m} \prod_{r=1}^{k}\left(z_{r}-1\right)^{u_{r}}
$$

Hence, if $\left|z_{r}-1\right|<1$ for all $r \in\{1, \ldots, k\}$, then

$$
\begin{aligned}
\sum_{w \in \mathbf{Z}_{+}^{k}} \mathbf{P}\left\{S_{n}=w\right\} z^{w}= & \left(1+\sum_{r=1}^{k} q_{r}\left(z_{r}-1\right)\right)^{n} \\
& \times \prod_{j=1}^{n}\left(1+\frac{\sum_{r=1}^{k}\left(p_{j, r}-q_{r}\right)\left(z_{r}-1\right)}{1+\sum_{r=1}^{k} q_{r}\left(z_{r}-1\right)}\right) \\
= & \sum_{|u| \leqslant n} a_{u}(q)\left(1+\sum_{r=1}^{k} q_{r}\left(z_{r}-1\right)\right)^{n-|u|} \prod_{r=1}^{k}\left(z_{r}-1\right)^{u_{r}} \\
= & \sum_{w \in \mathbf{Z}_{+}^{k}}\left(\sum_{|u| \leqslant n} a_{u}(q) \Delta^{u} M(w, n-|u|, q)\right) z^{w},
\end{aligned}
$$

giving the assertion.

The following two lemmas are devoted to some useful properties of the Krawtchouk polynomials.

Lemma 1. If $|w| \leqslant m$ and $|u| \leqslant m$, then

$$
K_{u}(w, m, q) M(w, m, q)=\frac{m ! q^{u} q_{0}^{|u|}}{u !(m-|u|) !} \Delta^{u} M(w, m-|u|, q) .
$$

Further, if $|w| \leqslant m$, then

$$
\begin{aligned}
& \sum_{|u| \leqslant m} K_{u}(w, m, q) z^{u}=\left(1-\sum_{r=1}^{k} q_{r} z_{r}\right)^{m-|w|} \prod_{r=1}^{k}\left(1+q_{0} z_{r}\right)^{w_{r}} \\
& \sum_{|u| \leqslant m} \widetilde{K}_{u}(w, m, q) z^{u}=\left(1-\sum_{r=1}^{k} q_{r} z_{r}\right)^{m-|w|} \prod_{r=1}^{k}\left(z_{r}+1-\sum_{s=1}^{k} q_{s} z_{s}\right)^{w_{r}} .
\end{aligned}
$$

P r o of. Using (4), it can be shown that, for $|u| \leqslant m$,

$$
\begin{aligned}
& \sum_{|w| \leqslant m} K_{u}(w, m, q) M(w, m, q) z^{w} \\
& \quad=\frac{m ! q^{u} q_{0}^{|u|}}{u !(m-|u|) !}\left(1+\sum_{r=1}^{k} q_{r}\left(z_{r}-1\right)\right)^{m-|u|} \prod_{r=1}^{k}\left(z_{r}-1\right)^{u_{r}}
\end{aligned}
$$


Relation (21) is proved by using (20). The proof of (22) is similar to that of (24). We now verify (23). For $|w| \leqslant m$, we have

$$
\begin{aligned}
& \left(1-\sum_{r=1}^{k} q_{r} z_{r}\right)^{m-|w|} \prod_{r=1}^{k}\left(z_{r}+1-\sum_{s=1}^{k} q_{s} z_{s}\right)^{w_{r}} \\
& =\left(1-\sum_{r=1}^{k} q_{r} z_{r}\right)^{m-|w|} \prod_{r=1}^{k}\left[\sum_{v_{r}=0}^{w_{r}}\left(\begin{array}{c}
w_{r} \\
v_{r}
\end{array}\right) z_{r}^{v_{r}}\left(1-\sum_{s=1}^{k} q_{s} z_{s}\right)^{w_{r}-v_{r}}\right] \\
& =\sum_{v \leqslant w}\left(1-\sum_{r=1}^{k} q_{r} z_{r}\right)^{m-|v|}\left[\prod_{r=1}^{k}\left(\begin{array}{c}
w_{r} \\
v_{r}
\end{array}\right)\right] z^{v} \\
& =\sum_{v \leqslant w} \sum_{|\widetilde{v}| \leqslant m-|v|} \frac{(m-|v|) !(-q)^{\tilde{v}}}{\tilde{v} !(m-|v|-|\tilde{v}|) !}\left[\prod_{r=1}^{k}\left(\begin{array}{c}
w_{r} \\
v_{r}
\end{array}\right)\right] z^{v+\tilde{v}} \\
& =\sum_{|u| \leqslant m} \sum_{v \leqslant u} \frac{(m-|v|) !(-q)^{u-v}}{(u-v) !(m-|u|) !}\left[\prod_{r=1}^{k}\left(\begin{array}{c}
w_{r} \\
v_{r}
\end{array}\right)\right] z^{u} .
\end{aligned}
$$

Here, the latter equality follows from the substitution $u=v+\widetilde{v}$. The lemma is proved.

Lemma 2. The relations

$$
\begin{aligned}
\sum_{|u| \leqslant m} & \sum_{|v| \leqslant m}\left[\sum_{|w| \leqslant m} M(w, m, q) K_{u}(w, m, q) K_{v}(w, m, q)\right] y^{u} z^{v} \\
= & {\left[1+q_{0}^{2} \sum_{r=1}^{k} q_{r} y_{r} z_{r}+q_{0}\left(\sum_{r=1}^{k} q_{r} y_{r}\right)\left(\sum_{r=1}^{k} q_{r} z_{r}\right)\right]^{m} } \\
= & \sum_{|u| \leqslant m} \sum_{|v|=|u|}\left(\sum_{w \leqslant u \wedge v} \frac{m !|u-w| ! q^{u+v-w} q_{0}^{|w+u|}}{w !(m-|u|) !(u-w) !(v-w) !}\right) y^{u} z^{v}
\end{aligned}
$$

are valid. Further, for $|u| \leqslant m$ and $|v| \leqslant m$,

$$
\sum_{|w| \leqslant m} M(w, m, q) K_{u}(w, m, q) \widetilde{K}_{v}(w, m, q)=\frac{\delta_{u, v} m ! q^{u} q_{0}^{|u|}}{u !(m-|u|) !},
$$

where $\delta_{u, v}$ is the Kronecker symbol.

P r o o f. Equality (25) is easily shown by using (22). A further computation of the right-hand side of (25) leads to

$$
\begin{aligned}
& \sum_{j=0}^{m}\left(\begin{array}{c}
m \\
j
\end{array}\right)\left(1+q_{0}^{2} \sum_{r=1}^{k} q_{r} y_{r} z_{r}\right)^{j}\left[q_{0}\left(\sum_{r=1}^{k} q_{r} y_{r}\right)\left(\sum_{r=1}^{k} q_{r} z_{r}\right)\right]^{m-j} \\
& =\sum_{j=0}^{m} \sum_{|w| \leqslant j} \sum_{|\tilde{u}|=m-j} \sum_{|\tilde{v}|=m-j} \frac{m !(m-j) ! q^{w+\tilde{u}+\tilde{v}} q_{0}^{2|w|+m-j}}{w !(j-|w|) ! \tilde{u} ! \tilde{v} !} y^{w+\tilde{u}} z^{w+\tilde{v}}
\end{aligned}
$$




$$
=\sum_{|\widetilde{u}| \leqslant m} \sum_{|\widetilde{v}|=|\widetilde{u}|} \sum_{|w| \leqslant m-|\tilde{u}|} \frac{m !|\tilde{u}| ! q^{w+\tilde{u}+\tilde{v}} q_{0}^{2|w|+|\tilde{u}|} y^{w+\tilde{u}} z^{w+\tilde{v}}}{w !(m-|\tilde{u}|-|w|) ! \tilde{u} ! \tilde{v} !}
$$

Here, the latter equality follows from the substitution $j=m-|\widetilde{u}|$. Substituting $\tilde{u}=u-w$ and $\widetilde{v}=v-w$ and observing that, here, $|v|=|\widetilde{v}|+|w|=$ $|\tilde{u}|+|w|=|u|,(26)$ is shown. Relation (27) is due to Tratnik [12, formula (3.9)] and can easily be verified by using (22) and (23). The proof is completed.

$\mathrm{R}$ e $\mathrm{m}$ a $\mathrm{r} \mathrm{k}$. For $k=2$, similar versions of (21), (22), and (27) can be found in Takeuchi and Takemura [11, Section 3].

P r o of of Proposition 1. Let $\left|z_{r}\right|<\frac{1}{2}$ for all $r \in\{1, \ldots, k\}$. By (3) and (23), we get

$$
\begin{aligned}
\sum_{|u| \leqslant n} a_{u}(q) z^{u}= & \left(1-\sum_{r=1}^{k} q_{r} z_{r}\right)^{n} \prod_{j=1}^{n}\left(1+\sum_{r=1}^{k} p_{j, r}\left[\frac{z_{r}+1-\sum_{s=1}^{k} q_{s} z_{s}}{1-\sum_{s=1}^{k} q_{s} z_{s}}-1\right]\right) \\
= & \sum_{|w| \leqslant n} \mathbf{P}\left\{S_{n}=w\right\}\left(1-\sum_{r=1}^{k} q_{r} z_{r}\right)^{n-|w|} \\
& \times \prod_{r=1}^{k}\left(z_{r}+1-\sum_{s=1}^{k} q_{s} z_{s}\right)^{w_{r}} \\
= & \sum_{|u| \leqslant n}\left(\sum_{|w| \leqslant n} \mathbf{P}\left\{S_{n}=w\right\} \widetilde{K}_{u}(w, n, q)\right) z^{u},
\end{aligned}
$$

giving (7). Using (3) we obtain (8):

$$
\begin{aligned}
& \sum_{1 \leqslant|u| \leqslant n}|u| a_{u}(q) z^{u}=\sum_{r=1}^{k} z_{r} \frac{\partial}{\partial z_{r}} \sum_{|u| \leqslant n} a_{u}(q) z^{u} \\
& =\sum_{r=1}^{k} z_{r}\left(\sum_{j=1}^{n} \frac{p_{j, r}-q_{r}}{1+\sum_{s=1}^{k}\left(p_{j, s}-q_{s}\right) z_{s}}\right) \prod_{j=1}^{n}\left(1+\sum_{s=1}^{k}\left(p_{j, s}-q_{s}\right) z_{s}\right) \\
& =-\sum_{j=1}^{n} \sum_{m=1}^{\infty}\left(\sum_{r=1}^{k}\left(q_{r}-p_{j, r}\right) z_{r}\right)^{m} \sum_{|u| \leqslant n} a_{u}(q) z^{u} \\
& =-\left[\sum_{|u| \geqslant 1} \frac{|u| !}{u !}\left(\sum_{j=1}^{n} \prod_{r=1}^{k}\left(q_{r}-p_{j, r}\right)^{u_{r}}\right) z^{u}\right]\left[\sum_{|u| \leqslant n} a_{u}(q) z^{u}\right] \\
& =-\sum_{1 \leqslant|u| \leqslant n}\left[\sum_{v<u} \frac{|u-v| !}{(u-v) !}\left(\sum_{j=1}^{n} \prod_{r=1}^{k}\left(q_{r}-p_{j, r}\right)^{u_{r}-v_{r}}\right) a_{v}(q)\right] z^{u} .
\end{aligned}
$$


Using the equality $\sum_{|u| \leqslant n} \mu_{(u)} z^{u} / u !=\prod_{j=1}^{n}\left(1+\sum_{r=1}^{k} p_{j, r} z_{r}\right)$ and (3), we get

$$
\begin{aligned}
\sum_{|u| \leqslant n} a_{u}(q) z^{u} & =\left(1-\sum_{r=1}^{k} q_{r} z_{r}\right)^{n} \prod_{j=1}^{n}\left(1+\frac{\sum_{r=1}^{k} p_{j, r} z_{r}}{1-\sum_{r=1}^{k} q_{r} z_{r}}\right) \\
& =\sum_{|v| \leqslant n} \frac{\mu_{(v)}}{v !} z^{v}\left(1-\sum_{r=1}^{k} q_{r} z_{r}\right)^{n-|v|} \\
& =\sum_{|v| \leqslant n} \sum_{|w| \leqslant n-|v|} \frac{(n-|v|) !(-q)^{w} \mu_{(v)}}{w !(n-|v|-|w|) ! v !} z^{v+w} \\
& =\sum_{|u| \leqslant n}\left(\sum_{v \leqslant u} \frac{(n-|v|) !(-q)^{u-v} \mu_{(v)}}{(u-v) !(n-|u|) ! v !}\right) z^{u},
\end{aligned}
$$

giving (9). Equality (10) follows from (3) and Cauchy's theorem. The proposition is proved.

For the proof of Theorem 2, we need the following three lemmas.

Lemma 3. Let $I_{0}(x)=\sum_{m=0}^{\infty}(x / 2)^{2 m} /(m !)^{2}$ be the modified Bessel function of the first kind and order $0, \beta(x)=I_{0}(x) e^{-x^{2} / 4}(x \in \mathbf{R})$. If $1 \leqslant|u| \leqslant n$ and $\gamma_{2}(r, q)>0$ for all $r \in\{1, \ldots, k\}$, then

$$
\begin{aligned}
&\left|a_{u}(q)\right| \leqslant \frac{n^{(n-|u|) / 2}|u|^{|u| / 2}}{(n-|u|)^{(n-|u|) / 2} u^{u}} \prod_{r=1}^{k} {\left[\left(\gamma_{2}(r, q)+\frac{n-|u|}{2 n} \gamma_{1}^{2}(r, q)\right)^{u_{r} / 2}\right.} \\
&\left.\times \beta\left(\sqrt{\frac{2 u_{r}^{2}(n-|u|) \gamma_{1}^{2}(r, q)}{|u|\left(2 n \gamma_{2}(r, q)+(n-|u|) \gamma_{1}^{2}(r, q)\right)}}\right)\right] .
\end{aligned}
$$

P r o o f. From (10), it follows that, for $1 \leqslant|u| \leqslant n$ and arbitrary $R \in(0, \infty)^{k}$,

$$
\left|a_{u}(q)\right| \leqslant \frac{1}{(2 \pi)^{k} R^{u}} \int_{0}^{2 \pi} \cdots \int_{0}^{2 \pi}\left|h\left(R_{1} e^{i x_{1}}, \ldots, R_{k} e^{i x_{k}}\right)\right| d x_{1} \cdots d x_{k},
$$

where $h(z)=\prod_{j=1}^{n}\left[1+\sum_{r=1}^{k}\left(p_{j, r}-q_{r}\right) z_{r}\right]$. Let $\alpha \in(0, \infty)$ be arbitrary. Using $\cos b_{1} \cos b_{2}+\sin b_{1} \sin b_{2}=\cos \left(b_{1}-b_{2}\right) \in[-1,1], 1+b_{1} \leqslant e^{b_{1}}$ for $b_{1}, b_{2} \in \mathbf{R}$, and Cauchy's inequality, we obtain

$$
\begin{aligned}
\left|h\left(R_{1} e^{i x_{1}}, \ldots, R_{k} e^{i x_{k}}\right)\right| & \\
=\frac{1}{\alpha^{n / 2}} \prod_{j=1}^{n}[\alpha & +2 \alpha \sum_{r=1}^{k}\left(p_{j, r}-q_{r}\right) R_{r} \cos x_{r} \\
& \left.\quad+\alpha \sum_{r=1}^{k} \sum_{s=1}^{k}\left(p_{j, r}-q_{r}\right)\left(p_{j, s}-q_{s}\right) R_{r} R_{s} \cos \left(x_{r}-x_{s}\right)\right]^{1 / 2}
\end{aligned}
$$




$$
\begin{gathered}
\leqslant \frac{1}{\alpha^{n / 2}} \exp \left(\frac{n(\alpha-1)}{2}-\alpha \sum_{r=1}^{k} \gamma_{1}(r, q) R_{r} \cos x_{r}\right. \\
\left.+\frac{\alpha}{2}\left(\sum_{r=1}^{k} \sqrt{\gamma_{2}(r, q)} R_{r}\right)^{2}\right) .
\end{gathered}
$$

Since

$$
\sum_{r=1}^{k} b_{r} \leqslant\left(\sum_{r=1}^{k} \sqrt{b_{r}}\right)^{2}, \quad\left(\sum_{r=1}^{k} \sqrt{b_{r}}\right)^{2}+\left(\sum_{r=1}^{k} \sqrt{b_{r}^{\prime}}\right)^{2} \leqslant\left(\sum_{r=1}^{k} \sqrt{b_{r}+b_{r}^{\prime}}\right)^{2}
$$

for $b, b^{\prime} \in[0, \infty)^{k}$, and $I_{0}(x)=\frac{1}{2 \pi} \int_{0}^{2 \pi} \exp (x \cos \nu) d \nu$ for $x \in \mathbf{R}$, we get

$$
\begin{aligned}
\left|a_{u}(q)\right| \leqslant & \frac{\prod_{r=1}^{k} \beta\left(\alpha \gamma_{1}(r, q) R_{r}\right)}{\alpha^{n / 2} R^{u}} \\
& \times \exp \left(\frac{n(\alpha-1)}{2}+\frac{1}{4}\left(\sum_{r=1}^{k} R_{r} \sqrt{2 \alpha \gamma_{2}(r, q)+\alpha^{2} \gamma_{1}^{2}(r, q)}\right)^{2}\right) .
\end{aligned}
$$

Finally, for $r \in\{1, \ldots, k\}$ and $\varepsilon>0$, set

$$
\alpha=\frac{n-|u|+\varepsilon}{n}, \quad R_{r}=\sqrt{\frac{2 u_{r}^{2}+\varepsilon}{|u|\left(2 \alpha \gamma_{2}(r, q)+\alpha^{2} \gamma_{1}^{2}(r, q)\right)}}
$$

and let $\varepsilon$ tend to zero. The proof is completed.

R e $\mathrm{m}$ a r k s. 1 . We have $0 \leqslant \beta(x)=\beta(|x|) \leqslant 1$ for $x \in \mathbf{R}$.

2. From Lemma 3, we get the inequality

$$
\left|a_{u}(q)\right| \leqslant \frac{n^{(n-|u|) / 2}|u|^{|u| / 2}}{(n-|u|)^{(n-|u|) / 2} 2^{|u| / 2} u^{u}} \prod_{r=1}^{k} \eta^{u_{r} / 2}(r, q)
$$

for $1 \leqslant|u| \leqslant n$, being also valid in the case of $\gamma_{2}(r, q)=0$ for some $r \in$ $\{1, \ldots, k\}$.

Lemma 4. The following inequality is valid:

$$
\frac{|u|^{|u|}}{u^{u}} \leqslant \exp \left(\sum_{r=2}^{k} u_{r}\right) \frac{|u| !}{u !} .
$$

P r o of. We may assume that $k \in\{2,3, \ldots\}$ and $u \in \mathbf{N}^{k}$. For $s \in$ $\{1, \ldots, k\}$, let $v_{s}=\sum_{r=1}^{s} u_{r}$. Using the inequalities

$$
\frac{b_{1}^{b_{1}}}{b_{2}^{b_{2}}\left(b_{1}-b_{2}\right)^{b_{1}-b_{2}}} \leqslant e \sqrt{b_{2}}\left(\begin{array}{l}
b_{1} \\
b_{2}
\end{array}\right), \quad b_{1} \in \mathbf{N}, b_{2} \in\left\{1, \ldots, b_{1}\right\}
$$


(see Roos [6, Lemma 3]) and $1+x \leqslant e^{x}, x \in \mathbf{R}$, we get

$$
\begin{aligned}
\frac{|u|^{|u|}}{u^{u}} & =\prod_{r=1}^{k-1} \frac{v_{r+1}^{v_{r+1}}}{v_{r}^{v_{r}} u_{r+1}^{u_{r+1}}} \leqslant e^{k-1} \sqrt{u_{2} \cdots u_{k}} \frac{|u| !}{u !} \\
& \leqslant \exp \left(\frac{1}{2} \sum_{r=2}^{k}\left(u_{r}+1\right)\right) \frac{|u| !}{u !} \leqslant \exp \left(\sum_{r=2}^{k} u_{r}\right) \frac{|u| !}{u !} .
\end{aligned}
$$

The lemma is proved.

Lemma 5. The following inequalities hold:

$$
\begin{aligned}
\left\|\Delta^{u} M(\cdot, m, q)\right\|_{1} & \leqslant\left(\frac{u ! m !}{(m+|u|) ! q^{u} q_{0}^{|u|}}\right)^{1 / 2} \\
& \leqslant\left(\frac{e^{|u|} u^{u} m^{m}}{(m+|u|)^{m+|u|} q^{u} q_{0}^{|u|}}\right)^{1 / 2} .
\end{aligned}
$$

P r o o f. Using (21), Cauchy's inequality, and (26), we obtain

$$
\begin{aligned}
& \left(\left\|\Delta^{u} M(\cdot, m, q)\right\|_{1} \frac{(m+|u|) ! q^{u} q_{0}^{|u|}}{u ! m !}\right)^{2} \\
& \quad \leqslant \sum_{|w| \leqslant m+|u|} M(w, m+|u|, q)\left[K_{u}(w, m+|u|, q)\right]^{2} \\
& =\frac{(m+|u|) ! q^{u} q_{0}^{2|u|}}{u ! m !} \sum_{w \leqslant u}\left[\prod_{r=1}^{k}\left(\begin{array}{c}
u_{r} \\
w_{r}
\end{array}\right)\right] \frac{|w| !}{w !} q^{w} q_{0}^{-|w|} \\
& \leqslant \frac{(m+|u|) ! q^{u} q_{0}^{2|u|}}{u ! m !} \sum_{w \leqslant u}\left[\prod_{r=1}^{k}\left(\begin{array}{c}
u_{r} \\
w_{r}
\end{array}\right)\right] \sum_{|v|=|w|} \frac{|w| !}{v !} q^{v} q_{0}^{-|v|} \\
& =\frac{(m+|u|) ! q^{u} q_{0}^{|u|}}{u ! m !}
\end{aligned}
$$

giving the first inequality of (30). The second inequality follows from (29).

$\mathrm{P}$ r o of of Theor e m 2. Let $I=\left\{r \in\{1, \ldots, k\} \mid q_{r} \leqslant e /\left(4 n q_{0}\right)\right\}$ and $I^{c}=\{1, \ldots, k\} \backslash I$. For $u \in \mathbf{Z}_{+}^{k}$, let $v(u)=\sum_{r \in I} u_{r} e_{r}$. To simplify notation, we write $v$ for $v(u)$. Using (30) and the inequality $1+x \leqslant e^{x}$, $x \in \mathbf{R}$, we get, for $|u| \leqslant n$,

$$
\begin{aligned}
\left\|\Delta^{u} M(\cdot, n-|u|, q)\right\|_{1} & \leqslant 2^{|v|}\left\|\Delta^{u-v} M(\cdot, n-|u|, q)\right\|_{1} \\
& \leqslant 2^{|v|}\left(\frac{e^{|u-v|}(u-v)^{u-v}(n-|u|)^{n-|u|}}{(n-|v|)^{n-|v|} q^{u-v} q_{0}^{|u-v|}}\right)^{1 / 2}
\end{aligned}
$$

and $n^{n-|v|}(u-v)^{u-v}\left[(n-|v|)^{n-|v|} u^{u}\right]^{-1} \leqslant 1$. Using

$$
\frac{|u|^{|u|}}{u^{u}} \leqslant\left(\frac{|u| !}{u !}\right)^{2}
$$


(see Roos [7, Lemma 4]), (2), and (28), we obtain

$$
\begin{aligned}
\left\|P^{S_{n}}-\mathscr{M}_{t}(n, q)\right\|_{1} \leqslant & \sum_{j=t+1}^{n} \sum_{|u|=j}\left|a_{u}(q)\right|\left\|\Delta^{u} M(\cdot, n-|u|, q)\right\|_{1} \\
\leqslant & \sum_{j=t+1}^{n} \sum_{|u|=j} \sqrt{\frac{|u|^{|u|} n^{n-|v|}(u-v)^{u-v}}{u^{u}(n-|v|)^{n-|v|} u^{u}}} \\
& \times \prod_{r \in I}(2 \eta(r, q))^{u_{r} / 2} \prod_{r \in I^{c}}\left(\frac{e \eta(r, q)}{2 n q_{r} q_{0}}\right)^{u_{r} / 2} \\
& \leqslant \frac{e^{(t+1) / 2}\left(\sum_{r=1}^{k} \sqrt{\delta(r, q)}\right)^{t+1}}{1-\sum_{r=1}^{k} \sqrt{e \delta(r, q)}}
\end{aligned}
$$

giving the assertion.

$\mathrm{P}$ r o of of $\mathrm{C}$ or o 11 a r y 1 . The assertions can easily be shown by using (14), the inequality $\left\|P^{S_{n}}-\mathscr{M}_{t}(n, q)\right\|_{1} \leqslant 1+\left\|\mathscr{M}_{t}(n, q)\right\|_{1}$, the remark after Theorem 2, and Bernoulli's inequality.

Proof of Corollary 2. For $r \in\{0, \ldots, k\}, A_{+}=\{j \in$ $\left.\{1, \ldots, n\} \mid p_{j, r} \geqslant \bar{p}_{r}\right\}$, and $A_{-}=\{1, \ldots, n\} \backslash A_{+}$, we have

$$
\begin{aligned}
\gamma_{2}(r, \bar{p}) & \leqslant\left(\sum_{j \in A_{+}}\left(p_{j, r}-\bar{p}_{r}\right)\right)^{2}+\left(\sum_{j \in A_{-}}\left(\bar{p}_{r}-p_{j, r}\right)\right)^{2} \\
& =\frac{1}{2}\left(\sum_{j=1}^{n}\left|p_{j, r}-\bar{p}_{r}\right|\right)^{2} \leqslant 2\left[n \bar{p}_{r}\left(1-\bar{p}_{r}\right)\right]^{2}
\end{aligned}
$$

and $\gamma_{2}(r, \bar{p}) \leqslant n \bar{p}_{r}\left(1-\bar{p}_{r}\right)$ (see Roos [6, proof of the remark in Section 1.2]). This leads to

$$
\gamma_{2}(r, \bar{p}) \min \left\{\frac{1}{n \bar{p}_{r}\left(1-\bar{p}_{r}\right)}, 1\right\} \geqslant \frac{1}{2}\left(\frac{\gamma_{2}(r, \bar{p})}{n \bar{p}_{r}\left(1-\bar{p}_{r}\right)}\right)^{2} .
$$

Using (17), we get

$$
\left\|P^{S_{n}}-\mathscr{M}(n, \bar{p})\right\|_{1} \geqslant \frac{1}{124(k+1)^{2}}\left(\sum_{r=0}^{k} \frac{\gamma_{2}(r, \bar{p})}{n \bar{p}_{r}\left(1-\bar{p}_{r}\right)}\right)^{2} .
$$

For a similar upper bound, we may suppose that $\bar{p}_{0} \geqslant 1 /(k+1)$, which can be achieved by interchanging of components. Hence, by Corollary 1 ,

$$
\left\|P^{S_{n}}-\mathscr{M}(n, \bar{p})\right\|_{1} \leqslant \frac{e k(k+1)}{2-\sqrt{3}} \sum_{r=0}^{k} \frac{\gamma_{2}(r, \bar{p})}{n \bar{p}_{r}\left(1-\bar{p}_{r}\right)},
$$

completing the proof.

$\mathrm{Pr}$ o of of $\mathrm{Pr}$ oposition 2. For $r \in\{1, \ldots, k\}$, let

$$
q_{r}=\frac{\bar{p}_{r}}{\sum_{s=1}^{k} \bar{p}_{s}}=\frac{p_{j, r}}{\sum_{s=1}^{k} p_{j, s}} \quad(j \in\{1, \ldots, n\}) .
$$


Then

$$
\begin{aligned}
\sum_{w \in \mathbf{Z}_{+}^{k}} \mathbf{P}\left\{S_{n}=w\right\} z^{w}=\prod_{j=1}^{n}\left[1+\left(\sum_{r=1}^{k} p_{j, r}\right)\left(\sum_{r=1}^{k} q_{r} z_{r}-1\right)\right] \\
=\sum_{j=0}^{n} \mathbf{P}\left\{T_{n}=j\right\}\left(\sum_{r=1}^{k} q_{r} z_{r}\right)^{j}=\sum_{|w| \leqslant n} \frac{|w| !}{w !} q^{w} \mathbf{P}\left\{T_{n}=|w|\right\} z^{w}
\end{aligned}
$$

and

$$
\sum_{w \in \mathbf{Z}_{+}^{k}} \mathscr{M}(n, \bar{p})(\{w\}) z^{w}=\sum_{|w| \leqslant n} \frac{|w| !}{w !} q^{w} \mathscr{B}\left(n, \sum_{r=1}^{k} \bar{p}_{r}\right)(\{|w|\}) z^{w} .
$$

The proof is easily completed.

For the proof of Theorem 3, we need the following two lemmas.

Lemma 6. Let $c_{3}=\sqrt{e} 2^{-1}(1+\sqrt{\pi / 2})$ and $\alpha \in(0, \infty)$. Then

$$
\begin{aligned}
& \int_{0}^{\pi / 2} \exp \left(-2 \alpha \sin ^{2} x\right) \sin ^{m} x d x \\
& \quad \leqslant \min \left\{\frac{\pi}{2}, c_{3} \pi\left(\frac{\max \{m, 1 / 3\}}{4 \alpha e}\right)^{(m+1) / 2}\right\} .
\end{aligned}
$$

P r o of. Shorgin [9, proof of Lemma 6] has shown that, for $m \in \mathbf{N}$,

$$
\int_{0}^{\pi / 2} \exp \left(-2 \alpha \sin ^{2} x\right) \sin ^{m} x d x \leqslant \frac{\pi \sqrt{e}}{2}\left(1+\sqrt{\frac{\pi}{2 m}}\right)\left(\frac{m}{4 \alpha e}\right)^{(m+1) / 2} \text {. }
$$

Using the inequality $2 x / \pi \leqslant \sin x$ for $x \in[0, \pi / 2]$, we get, in the case $m=0$,

$$
\int_{0}^{\pi / 2} \exp \left(-2 \alpha \sin ^{2} x\right) d x \leqslant \int_{0}^{\pi / 2} \exp \left(-\frac{8}{\pi^{2}} \alpha x^{2}\right) d x \leqslant c_{3} \pi\left(\frac{1 / 3}{4 \alpha e}\right)^{1 / 2} .
$$

This gives one part of (32). The remaining part is obvious. The lemma is proved.

Lemma 7. Let $c_{3}$ be as in Lemma 6, I $\subseteq\{1, \ldots, k\}$, and $I^{c}=$ $\{1, \ldots, k\} \backslash$ I. If $u \neq 0$, then

$$
\begin{aligned}
\left\|\Delta^{u} M(\cdot, m, q)\right\|_{\infty} \leqslant & \left(\frac{m}{m+|u|}\right)^{m / 2}\left(\prod_{r \in I}(4 e)^{u_{r} / 2}\right) \\
& \times \prod_{r \in I^{c}}\left[\frac{c_{3}}{\sqrt{e}}\left(\frac{\max \left\{u_{r}, 1 / 3\right\}}{(m+|u|) q_{r} q_{0}}\right)^{\left(u_{r}+1\right) / 2}\right] .
\end{aligned}
$$

P r o of. By (20) and Cauchy's theorem, we have

$$
\left\|\Delta^{u} M(\cdot, m, q)\right\|_{\infty} \leqslant \frac{1}{(2 \pi)^{k}} \int_{-\pi}^{\pi} \cdots \int_{-\pi}^{\pi} H(x) d x_{1} \cdots d x_{k},
$$


where, for $x \in[-\pi, \pi]^{k}$,

$$
\begin{aligned}
H(x)= & \left|1+\sum_{r=1}^{k} q_{r}\left(e^{i x_{r}}-1\right)\right|^{m} \prod_{r=1}^{k}\left|e^{i x_{r}}-1\right|^{u_{r}} \\
= & \left(q_{0}^{2}+2 q_{0} \sum_{r=1}^{k} q_{r} \cos x_{r}+\sum_{r=1}^{k} \sum_{s=1}^{k} q_{r} q_{s} \cos \left(x_{r}-x_{s}\right)\right)^{m / 2} \\
& \times 2^{|u|} \prod_{r=1}^{k}\left|\sin \left(\frac{x_{r}}{2}\right)\right|^{u_{r}} \\
\leqslant & 2^{|u|}\left(1-4 q_{0} \sum_{r=1}^{k} q_{r} \sin ^{2}\left(\frac{x_{r}}{2}\right)\right)^{m / 2} \prod_{r=1}^{k}\left|\sin \left(\frac{x_{r}}{2}\right)\right|^{u_{r}} .
\end{aligned}
$$

Here, we used the relations $1-\cos b_{1}=2 \sin ^{2}\left(b_{1} / 2\right)$ and $\cos b_{1} \cos b_{2}+$ $\sin b_{1} \sin b_{2}=\cos \left(b_{1}-b_{2}\right) \leqslant 1$ for $b_{1}, b_{2} \in \mathbf{R}$. Hence

$$
\left\|\Delta^{u} M(\cdot, m, q)\right\|_{\infty} \leqslant \frac{2^{|u|+k}}{\pi^{k}} f_{u, m}(q),
$$

where, for $\varepsilon \in[0, \infty)$,

$$
f_{u, \varepsilon}(q)=\int_{0}^{\pi / 2} \cdots \int_{0}^{\pi / 2}\left(1-4 q_{0} \sum_{r=1}^{k} q_{r} \sin ^{2} x_{r}\right)^{\varepsilon / 2} \prod_{r=1}^{k} \sin ^{u_{r}} x_{r} d x_{1} \cdots d x_{k} .
$$

For $\varepsilon \in(0, \infty)$ and arbitrary $\alpha \in(0, \infty)$,

$$
\begin{aligned}
f_{u, \varepsilon}(q) & \leqslant \frac{e^{\varepsilon(\alpha-1) / 2}}{\alpha^{\varepsilon / 2}} \prod_{r=1}^{k}\left(\int_{0}^{\pi / 2} \exp \left(-2 \alpha \varepsilon q_{r} q_{0} \sin ^{2} x_{r}\right) \sin ^{u_{r}} x_{r} d x_{r}\right) \\
& \leqslant \pi^{k} g(\alpha) 2^{-\# I} \prod_{r \in I^{c}}\left[c_{3}\left(\frac{\max \left\{u_{r}, 1 / 3\right\}}{4 \varepsilon q_{r} q_{0} e}\right)^{\left(u_{r}+1\right) / 2}\right],
\end{aligned}
$$

where $g(\alpha)=\alpha^{-(\varepsilon+\nu) / 2} e^{\varepsilon(\alpha-1) / 2}, \nu=\sum_{r \in I^{c}}\left(u_{r}+1\right)$, and \#I denotes the number of elements of $I$. Here, we used the inequality $1+b \leqslant e^{b}$ for $b \in \mathbf{R}$ and (32). Now, $g(\alpha)$ attains its minimum at $\alpha_{0}=(\varepsilon+\nu) / \varepsilon$. Substituting $\alpha=\alpha_{0}$, we obtain

$$
f_{u, \varepsilon}(q) \leqslant \pi^{k} \frac{\varepsilon^{\varepsilon / 2}(\varepsilon+|u|)^{\nu / 2}}{(\varepsilon+\nu)^{(\varepsilon+\nu) / 2}} 2^{-\# I} \prod_{r \in I^{c}}\left[c_{3}\left(\frac{\max \left\{u_{r}, 1 / 3\right\}}{4(\varepsilon+|u|) q_{r} q_{0}}\right)^{\left(u_{r}+1\right) / 2}\right] .
$$

Application of the inequality $1+b \leqslant e^{b}$ leads to the desired result in the case $m \neq 0$. For $m=0$, the assertion is proved by letting $\varepsilon \downarrow 0$ in the inequalities above.

$\mathrm{Pr}$ o of of $\mathrm{Th}$ e o r e m 3. Let $c_{3}$ be as in Lemma 6, $I=\{r \in$ $\left.\{1, \ldots, k\} \mid q_{r} \leqslant 3 /\left(4 e n q_{0}\right)\right\}$, and $I^{c}=\{1, \ldots, k\} \backslash I$. Using (28), (33), (31), 
the inequality $\left(\max \left\{m, \frac{1}{3}\right\}\right)^{m+1} / m^{m} \leqslant 3^{m-1}$, and

$$
\prod_{r \in I^{c}} \frac{1}{n q_{r} q_{0}}=\left(\frac{3}{4 e}\right)^{\# I} \prod_{r=1}^{k} \min \left\{\frac{1}{n q_{r} q_{0}}, \frac{4 e}{3}\right\}
$$

we obtain (19):

$$
\begin{aligned}
& \left\|P^{S_{n}}-\mathscr{M}_{t}(n, q)\right\|_{\infty} \leqslant \sum_{j=t+1}^{n} \sum_{|u|=j}\left|a_{u}(q)\right|\left\|\Delta^{u} M(\cdot, n-|u|, q)\right\|_{\infty} \\
& \leqslant \sum_{j=t+1}^{n} \sum_{|u|=j} \frac{|u| !}{u !}\left(\prod_{r \in I}(2 e \eta(r, q))^{u_{r} / 2}\right) \prod_{r \in I^{c}}\left(\frac{c_{3}}{\sqrt{3 e n q_{r} q_{0}}}\left(\frac{3 \eta(r, q)}{2 n q_{r} q_{0}}\right)^{u_{r} / 2}\right) \\
& \quad \leqslant 3^{(t+1) / 2} c_{2}^{k}\left(\prod_{r=1}^{k} \min \left\{\frac{1}{n q_{r} q_{0}}, \frac{4 e}{3}\right\}\right)^{1 / 2} \frac{\left(\sum_{r=1}^{k} \sqrt{\zeta(r, q)}\right)^{t+1}}{1-\sum_{r=1}^{k} \sqrt{3 \zeta(r, q)}},
\end{aligned}
$$

completing the proof.

Acknowledgment. I thank Lutz Mattner and the referee for their useful remarks, which led to an improvement of the paper.

\section{REFERENCES}

1. Barbour A.D. Stein's method and Poisson process convergence. - J. Appl. Probab., 1988 , spec. v. $25 \mathrm{~A}$, p. $175-184$.

2. Deheuvels $P$., Pfeifer $D$. Poisson approximations of multinomial distributions and point processes. - J. Multivariate Anal., 1988, v. 25, p. 65-89.

3. Ehm $W$. Binomial approximation to the Poisson binomial distribution. - Statist. Probab. Lett., 1991, v. 11, p. 7-16.

4. Griffiths R. C. Orthogonal polynomials on the multinomial distribution. - Austral. J. Statist., 1971, v. 13, p. 27-35; Corrigenda: 1972, v. 14, p. 270.

5. Loh W.-L. Stein's method and multinomial approximation. - Ann. Appl. Probab., 1992 , v. 2 , p. $536-554$.

6. Roos B. Binomial approximation to the Poisson binomial distribution: The Krawtchouk expansion. - Теория вероятн. и ее примен., 2000, т. 45, в. 2, с. 328344.

7. Roos B. Metric multivariate Poisson approximation of the generalized multinomial distribution. - Теория вероятн. и ее примен., 1998, т. 43, с. 404-413.

8. Roos $B$. On the rate of multivariate Poisson convergence. - J. Multivariate Anal., 1999 , v. 69 , p. $120-134$.

9. Шоргия С. Я. Аппроксимация обобщенного биномиального распределения. - Теория вероятн. и ее примен., 1977, т. 22, в. 4, с. 867-871.

10. Szegö G. Orthogonal Polynomials. Providence, RI: Amer. Math. Soc., 1975, 432 p.

11. Takeuchi $K$., Takemura A. On sum of $0-1$ random variables. II. Multivariate case. Ann. Inst. Statist. Math., 1987, v. 39, p. 307-324.

12. Tratnik M. V. Multivariable Meixner, Krawtchouk, and Meixner-Pollaczek polynomials. - J. Math. Phys., 1989, v. 30, p. 2740-2749. 